\title{
Treatment of Narcolepsy with Cataplexy - An Overview of the Disease and a Report on Sodium Oxybate Dosage and Prescribing Information
}

\author{
a report by \\ Michael J Thorpy, ${ }^{1}$ Geert Mayer ${ }^{2}$ and John Shneerson ${ }^{3}$
}

1. Sleep-Wake Disorders Center, Department of Neurology, Montefiore Medical Center, New York;

2. Medical and Psychological Faculties, Philipps University, Marburg; 3. Respiratory Support and Sleep Centre, Papworth Hospital, Papworth Everard

DOI:10.17925/ENR.2008.03.01.84

Narcolepsy with cataplexy is a chronic sleep disorder primarily characterised by excessive daily sleepiness (EDS) and cataplexy. ${ }^{1}$ Two forms of narcolepsy have been identified: the majority of patients (60-70\% according to studies $\left.{ }^{2}\right)$ suffer from narcolepsy with cataplexy, whereas the others do not appear to present symptoms of cataplexy. ${ }^{3}$ To date, it is still considered unusual to pass rapidly from a wakeful state into rapid eye movement (REM) sleep and to experience distinct REM sleep events. ${ }^{1}$ The aetiology of narcolepsy is unknown; however, human and animal data suggest that narcolepsy with cataplexy is associated with abnormalities of the hypocretin system, with a loss of hypocretin neurons in the hypothalamus. ${ }^{3}$ The hypocretin system is a major excitatory system with neural connections on the monoaminergic and cholinergic systems, and therefore has a significant effect on vigilance states. ${ }^{4}$

\section{Clinical Features of Narcolepsy}

EDS is usually the first symptom that appears. It is characterised by multiple episodes of naps or lapses into sleep during daytime. ${ }^{1}$ Sleepiness is more likely to occur in mundane situations, although it may happen in unusual situations such as eating, walking or driving, and may lead to sudden and irresistible sleep attacks.

Cataplexy is the only specific symptom of narcolepsy. ${ }^{5}$ Typically, it is a sudden loss of bilateral muscle tone triggered by strong emotions. These emotions are usually positive, such as laughter, pride and surprise, but can also be negative, such as anger. ${ }^{1}$ Startling stimuli, stress, physical fatigue or sleepiness may also be factors that exacerbate cataplexy. ${ }^{6}$ Cataplexy can be localised or progressively include all skeletal muscle groups, with the patient ultimately collapsing to the ground. The most commonly affected areas are the knees, face and neck. The respiratory muscles are never affected. ${ }^{1}$ As cataplexy may also vary in frequency and severity, milder forms are more difficult to diagnose than more severe cases. As the only clinical symptom, mild sensations of weakness may pass completely unnoticed. Therefore, it can be relevant to probe the patient or her or his family members for experiences of head drop, facial sagging, jaw weakness, slurred speech or buckling of the knees. The duration of this symptom may also vary: it ranges from a couple of seconds to several minutes, always with a complete recovery. ${ }^{1}$

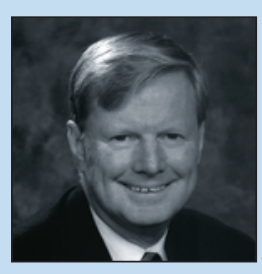

Michael I Thorpy is a Professor of Neurology at Albert Einstein College of Medicine and Director of the Sleep-Wake Disorders Center at Montefiore Medical Center, New York. $\mathrm{He}$ is the founder and Past Director of the National Sleep Foundation (NSF)'s National Narcolepsy Registry, and has served as Chairman of the Sleep Section of the American Academy of Neurology (AAN).

E: thorpy@aecom.yu.edu
The other associated symptoms are hypnagogic hallucinations and sleep paralysis, which is more likely transient, and disturbed nocturnal sleep. When the latter feature is present, it appears not to have a tendency to improve over time. ${ }^{7}$ Hypnagogic hallucinations may be visual, tactile, auditory or multisensory events. They occur at the transition from wakefulness to sleep, or from sleep to wakefulness. These hallucinations may contain dream elements. Typically, sleep paralysis is the inability to move during these same transition periods between sleep and wakefulness. Fragmented nocturnal sleep is characterised by frequent awakenings during the night. ${ }^{2}$ This disrupted nocturnal sleep occurs in one-third of patients. ${ }^{8}$ This symptom has long been underestimated, although today it is recognised as being a major indication in many patients. The current tetrad, i.e. EDS, cataplexy, hallucinations and sleep paralysis, has become a 'pentad' of symptoms for narcolepsy.

\section{Impact on Quality of Life}

Narcolepsy has a clear impact on quality of life (QoL). In a UK study, ${ }^{9}$ the greatest effect was on role limitation due to physical problems. The amount of time that patients spend on work or other activities is restricted. Leisure activities are also curtailed because patients tend to try to avoid potentially embarrassing situations. Narcolepsy also affects schooling, due to a difficulty to maintain concentration, as well as daily tasks such as cooking or taking care of children. It has been suggested that an accumulation of worries and concerns can be detrimental to emotional health: more than $50 \%$ of narcoleptics are depressed to some extent. Therefore, this pathology is extremely incapacitating.

\section{Epidemiology and Predisposing Factors}

The prevalence of narcolepsy is estimated to be around 25 per 100,000 in Caucasian populations. ${ }^{7}$ It can manifest at any age, but in most cases the symptoms appear between 10 and 25 years of age, ${ }^{10}$ and there are two peaks for onset: 15 and 36 years. ${ }^{8}$ Slightly more men than women are affected (relative risk 1.6/1). Approximately $1-4 \%$ of cases of narcolepsy have an affected family member. ${ }^{5}$ When narcolepsy runs in families, it typically shows an autosomal-dominant mode of inheritance. The vast majority of patients have sporadic narcolepsy (non-familial), but genetic factors are still important: the risk for a first-degree relative of a patient with narcolepsy is $1-2 \%$. A major contribution comes from environmental factors, and studies show that $25-31 \%$ of monozygotic twins are concordant for the disease. About $90 \%$ of patients suffering from narcolepsy with cataplexy have low or undetectable levels of hypocretin-1-orexin A in the cerebrospinal fluid (CSF). ${ }^{6}$ These low levels are relatively specific for narcolepsy with cataplexy, but are also seen in other central nervous system (CNS) pathologies such as the Guillain-Barré syndrome. There is also a strong but incomplete correlation between narcolepsy with cataplexy and the human leukocyte antigen (HLA) 
subtype $D Q B 1{ }^{*} 0602$, even if this subtype is common in the general population (20\% in the US). It is neither specific nor sensitive for narcolepsy. ${ }^{6}$ Hypocretin deficiency in the CSF is highly specific for HLA $D Q B 1 * 0602$ positive narcolepsy with cataplexy. ${ }^{5}$

\section{Diagnostic Criteria of Narcolepsy with Cataplexy}

In the International Classification of Sleep Disorders (ICSD), the diagnostic criteria include the following: ${ }^{1}$

- complaints of EDS occurring almost daily for at least three months;

- a clear history of cataplexy, defined as sudden and transient episodes of loss of muscle tone triggered by emotions;

- whenever possible, a diagnosis should be confirmed by nocturnal polysomnography followed by a multiple sleep latency test (MSLT). The mean sleep latency on MSLT should be less than or equal to eight minutes, and two or more sleep-onset REM periods should be observed following sufficient nocturnal sleep (minimum six hours) during the night prior to the test. Alternatively, hypocretin-1 levels in the CSF are less than or equal to $110 \mathrm{pg} / \mathrm{ml}$ or one-third of mean normal values; and

- the hypersomnia is not better explained by another sleep disorder, medical or neurological disorder, mental disorder, medication use or substance use disorder.

\section{Differential Diagnosis}

There are many other pathologies associated with hypersomnia, such as sleep apnoea syndrome, idiopathic hypersomnia, recurrent hypersomnia, chronic sleep deprivation and hypersomnia associated with depression. ${ }^{8}$

\section{Treatment Recommendations}

\section{The European Federation of Neurological Societies Guidelines on Management of Narcolepsy ${ }^{7}$}

Recommendations for the treatment of EDS: "First-line pharmacological treatment is modafinil, $100-400 \mathrm{mg} / \mathrm{d}$, given in two doses, one in the morning and one early in the afternoon (level A evidence). Of note, there is a growing practice in the US, based on level A evidence, of using sodium oxybate as a first-line therapy for EDS. In severe cases, a combination of modafinil and sodium oxybate appears to be beneficial. Behaviour treatment measures are always advisable: naps during the day (level B evidence)." Recommendations for the treatment of cataplexy: "Based on level A evidence, first-line pharmacological treatment of cataplexy is sodium oxybate at a dose starting at $4.5 \mathrm{~g} / \mathrm{night}$. The dose may be increased to a maximum of $9 \mathrm{~g} /$ night. Most patients will start to feel better after the first few days, but optimal response at any given dose may take as long as eight to 12 weeks. Second-line are antidepressants: tricyclics, particularly clomipramine $(10-75 \mathrm{mg})$, are the most anticataplectic, but with the drawback of anticholinergic adverse events. Other drugs are used but lack published clinical evidence (e.g norepinephrine/serotonine re-uptake inhibitor)."

\section{The American Academy of Sleep Medicine Guidelines ${ }^{11}$}

The recommendations for the treatment of narcolepsy are:

- treatment objectives should include control of sleepiness and other sleep-related symptoms when present (standard);

- modafinil is effective for the treatment of daytime sleepiness due to narcolepsy (standard);

- sodium oxybate is effective for the treatment of cataplexy, daytime sleepiness and disrupted sleep due to narcolepsy (standard);

- sodium oxybate may be effective for the treatment of hypnagogic hallucinations and sleep paralysis (option);

- amphetamine, methamphetamine, dextroamphetamine and methylphenidate are effective for the treatment of EDS due to narcolepsy (moderate guideline);

- tricyclic antidepressants, selective serotonin re-uptake inhibitors (SSRIS), venlafaxine and reboxetine may be effective treatment for cataplexy (moderate guideline); and

- scheduled naps can be beneficial to combat sleepiness (moderate guideline).

\section{Sodium Oxybate - An Overview of Clinical Data}

\section{Mode of Action}

Sodium oxybate was discovered in the 1960s as part of a search for a clinically useful analogue of the neurotransmitter $\gamma$-aminobutyric acid (GABA). ${ }^{12}$ It is a normal component of the nervous system, where it is referred to as $\gamma$-hydroxybutyrate (GHB). Binding studies indicate that there are at least two types of GHB-specific receptors: those with high affinity and those with low affinity for GHB. ${ }^{12}$ A GHB-specific high-affinity receptor (GHBR) has been identified through which some of the clinical effects may be mediated. ${ }^{13}$ In addition, at pharmacological concentration levels sodium oxybate binds $G A B A_{B}$ receptors, thus acting as a $G A B A_{B}$ agonist.13 Results from animal studies indicate that $G A B A_{B}$ receptors play an important role in the effects produced by pharmacological doses of sodium oxybate. ${ }^{13}$ The roles of GHB and GABA in mediating the behavioural effects of sodium oxybate have been explored using animal models. In such studies, selective antagonists for the $G_{A B A_{B}}$ receptor block the sedative effect of sodium oxybate ${ }^{13}$ and reduce the induction of non-REM sleep (including slow-wave sleep). Also, sodium oxybate has further effects on neurotransmitter levels, modulating the activity of dopaminergic, noradrenergic and serotonergic neurons. ${ }^{14}$ In particular, the $G_{A B A}$ receptor regulates the noradrenergic neurons of the locus coeruleus, which is the major adrenergic centre of the brain. This centre is involved in both the regulation of the sleep-wake cycle and behavioural states linked to attention and vigilance.

\section{Clinical Trials}

Pharmacokinetic studies have evaluated sodium oxybate for safety and efficacy in a total of 755 individuals, comprising 630 patients and 125 healthy subjects. Three placebo-controlled, randomised clinical trials evaluated the efficacy of sodium oxybate in the treatment of various narcolepsy symptoms. Four open-label studies - primarily designed as longitudinal safety studies - also assessed the effect of sodium oxybate on various symptoms of narcolepsy, including cataplexy, EDS and disturbed night-time sleep. Considerable data have also shown the beneficial impact of sodium oxybate therapy on QoL. The purpose of this article is to highlight the main outcome of these studies. Further details are to be found in the original published articles.

\section{Efficacy of Sodium Oxybate for the} Treatment of Cataplexy

The safety and efficacy of sodium oxybate for the treatment of cataplexy associated with narcolepsy was established in two randomised, doubleblind, placebo-controlled studies (Trials GHB-2 and SXB-15) and the openlabel extension to Trial GHB-2 (Trial GHB-3):

- Data from 136 patients in Trial GHB-2 showed that sodium oxybate produces a dose-related decrease in the total number of 
cataplexy attacks when taken for four weeks in equally divided nightly doses. ${ }^{15}$ Compared with placebo, sodium oxybate reduced the number of cataplexy attacks, with median reductions of $49 \%$ $(p=0.0529)$ and $69 \%(p=0.0008)$ in the sodium oxybate 6 and $9 g$ dose groups, respectively.

- In Trial SXB-15, 228 patients presented a weekly median of 18.5 cataplexy attacks across all dose groups. After eight weeks, the median decreases in cataplexy for patients randomised to sodium oxybate $4.5,6$ and $9 g$ were $57 \%(p<0.001), 65 \%(p<0.001)$ and $84.7 \%(p<0.001)$ versus placebo, respectively. ${ }^{16}$ As in Trial GHB-2, the greatest reduction occurred during the first four weeks of treatment; however, additional improvements were noted over the entire eight weeks of blinded treatment.

- In Trial GHB-3 (the extension to Trial GHB-2), 118 of the patients enrolled in Trial GHB-2 received open-label treatment for up to 12 months, beginning treatment with $6 \mathrm{~g}$ administered as two evenly divided nightly doses, with dose adjustments allowed at two-week intervals. In general, the number of cataplectic attacks continued to decrease over the first three months of this trial (total therapy of four months) and subsequently remained stable for the remainder of the trial. There was no evidence that tolerance developed to the effects of sodium oxybate. ${ }^{17}$

- Trial SXB-21 was a multicentre, randomised, double-blind, placebocontrolled study involving 55 patients who had been taking sodium oxybate on an open-label basis at doses of 3-9g nightly for seven to 44 months (mean 21 months). Cessation of long-term sodium oxybate therapy resulted in a gradual return of cataplexy in placebo patients over a two-week period $(p<0.001)$, whereas no median change was observed in the sodium-oxybate-treated patients. These data indicate that sodium oxybate remained effective after an average prior treatment period of 21 months. ${ }^{18}$

\section{Efficacy of Sodium Oxybate in Treating Excessive Daytime Sleepiness}

\section{Trial SXB-20}

This 10-week pilot study enrolled 25 patients suffering from narcolepsy, with dose escalation occurring in 21 patients. Stimulants for treatment of daytime sleepiness were continued throughout the trial. Effects on EDS were measured by the Maintenance of Wakefulness Test (MWT) and the Epworth Sleepiness Score (ESS). Sleep latency increased significantly in a dose-dependent manner. ${ }^{19}$ A progressive dose- and time-dependent increase in ESS was seen, becoming significant at week four.

\section{Trial SXB-15}

This eight-week trial assessed the efficacy of sodium oxybate (4.5, 6 and 9g) in 228 patients. Prior to randomisation and baseline assessment, subjects entered a two-week lead-in period during which narcolepsy symptoms and adverse events were recorded in daily diaries while maintaining treatment with their standard stimulant and anticataplexy treatments (ACTs). Patients were then gradually withdrawn from antidepressants over a period of 21 days. This was followed by a five- to 18-day wash-out period, depending on the half-life of the patient's prior, discontinued activated clotting time (ACT). Concomitant use of stimulants was permitted to continue at stable doses. After assignment to the final dose group - the double-blinded administration of placebo or particular sodium oxybate dose group patients entered a 14-21-day baseline assessment period during which they received placebo in a single-blinded fashion. ${ }^{20}$ After eight weeks of treatment, sodium oxybate produced a dose-related decrease in the ESS.
The median changes from baseline in ESS scores were $-1,-2$ and -5 with sodium oxybate doses of $4.5,6$ and $9 \mathrm{~g}$, respectively. Following treatment with sodium oxybate, a quartile analysis indicated that at least $25 \%$ of patients in the $6 \mathrm{~g}$ group fell into the intermediate, 'moderately sleepy' range (ESS 10-13), and more than $25 \%$ of patients in the $9 \mathrm{~g}$ group had normal scores (ESS $<10)$. These results indicated incremental decreases in EDS with sodium oxybate beyond those attributable to the stable dosing of stimulant medications that were maintained throughout the trial. Measured objectively using MWT, EDS also improved in patients taking $9 \mathrm{~g}$ of sodium oxybate, with a 10-minute increase in the MWT sleep latency $(p<0.001$ versus placebo). ${ }^{20}$ In addition, sodium oxybate treatment was associated with a decrease in the median number of inadvertent naps per week, which was significant at the 6 and $9 g$ doses compared with placebo $(p<0.001$ and $p=0.002$, respectively).

\section{Trial SXB-22}

This eight-week randomised, double-blind, double-dummy, placebocontrolled, parallel-group, multicentre trial compared the effects of orally administered sodium oxybate, either as monotherapy or together with modafinil, with placebo in 222 patients. Switching patients from modafinil to sodium oxybate monotherapy did not result in any significant change from baseline in MWT sleep latency. This indicates that sodium oxybate monotherapy was able to maintain the benefits achieved by prior treatment with modafinil. When sodium oxybate and modafinil were combined, the median MWT sleep latency was significantly increased by 2.7 minutes over baseline $(p<0.001)$, and the median change from baseline was significantly different from that seen in patients receiving placebo $(p<0.001) .{ }^{21}$

\section{Efficacy of Sodium Oxybate in Treating \\ Disrupted Nocturnal Sleep}

Despite continued administration of daytime stimulants, increases in slow-wave sleep and delta power were confirmed over eight weeks of treatment in the large-scale Trial SXB-15.22 Delta power is a feature of all stages of non-REM sleep, and is typically reduced in narcolepsy. The increase in slow-wave sleep was accompanied by a reduction in stage 1 sleep and an improved continuity of night-time sleep, with a significant reduction in the number of night-time awakenings at the 6 and $9 \mathrm{~g}$ doses. The duration of total sleep time was increased over eight weeks and reached significance at the $9 \mathrm{~g}$ dose.

\section{Effects of Sodium Oxybate on Quality of Life}

- An additional study has focused on QoL over six months (Trial SXB-6). This open-label, multicentre trial was performed to investigate the effect of prolonged sodium oxybate treatment on QoL, as measured at baseline and at six months by the Short Form (SF)-36 survey questionnaire. A statistically significant improvement $(p<0.05)$ was seen in seven of the eight domains measured by the SF-36 questionnaire, namely physical functioning, limitations due to physical functioning, general health, vitality, social functioning, limitations due to emotional problems and mental health.23

- The impact of therapy with sodium oxybate on QoL was also assessed in Trial SXB-15 with the validated Functional Outcomes of Sleep Questionnaire (FOSQ). The FOSQ is a condition-specific survey that analyses the effect of excessive sleepiness disorders on functional outcomes as they relate to daily behaviours and QoL. Analysis with regard to the FOSQ revealed that compared with placebo, nightly administration of sodium oxybate (6 or 9g) significantly improved many aspects of daily function related to the 
QoL of patients with narcolepsy. ${ }^{24}$ Improvements were seen in the activity level, vigilance, general productivity and social outcomes subscales.

\section{Clinical Safety Profile of Sodium Oxybate}

- Sodium oxybate has demonstrated a manageable safety profile in placebo-controlled and open-label clinical trials. In these studies, sodium oxybate was administered at 3-9g/night in two divided doses to 781 patients, typically in conjunction with stimulants for EDS. Combined data from these patients confirm the safety of sodium oxybate seen in the total population treated to date. The most commonly observed adverse events associated with the use of sodium oxybate in these trials were nausea, dizziness, headache, somnolence, vomiting, urinary incontinence and sleep walking. ${ }^{25}$ The overall rate of discontinuation due to adverse events in these trials was $14.7 \%$ with sodium oxybate and $5.8 \%$ with placebo. ${ }^{25}$ The most frequent reasons for discontinuation were nausea and psychiatric or nervous system events.

- The profile of adverse events seen over 12 months was similar to that seen in short-term studies, and the most commonly reported adverse events considered to be related to sodium oxybate therapy were headache $(33 \%)$, nausea $(28 \%)$, dizziness $(27 \%)$, somnolence $(20 \%)$ and enuresis $(8.5 \%) .{ }^{17}$ Of these, only dizziness showed any relationship with dose $(p<0.05)$. Serious adverse events were rare, and there were no deaths during the study.

\section{Sodium Oxybate}

\section{Prescribing Information and Practical} Considerations for the Clinicians

Sodium oxybate is the first European Medicines Agency (EMEA)-approved product proved to be effective for the treatment of all of the core symptoms of narcolepsy in adult patients with cataplexy. This approval is based on four multicentre, randomised, double-blind, placebocontrolled, parallel-group trials that demonstrated the efficacy and safety of the nightly administration of sodium oxybate for the treatment of daytime narcolepsy symptoms. ${ }^{12}$ Prescribing information and practical considerations for the clinicians are summarised below, using the recommendations of the product monograph and the personal experience of experts (Barcelona, 18-19 April 2008). As some statements may go beyond the currently approved indication in Europe or other countries, clinicians should consult their national legislation for the appropriate use of sodium oxybate. Nevertheless, these communications represent personal opinions by sleep specialists based on their experience in the use of sodium oxybate in more than 100 patients.

\section{Using Sodium Oxybate}

A first dose of sodium oxybate should be taken orally after getting into bed and a second dose taken between 2.5 and four hours after the first. Both doses of sodium oxybate should be prepared at the same time before retiring to bed. ${ }^{26}$ Sodium oxybate is provided with a graduated measuring syringe and two dosing cups, each with a child-resistant cap. Each measured dose of sodium oxybate is dispensed into the dosing cup and diluted with $60 \mathrm{ml}$ of water prior to ingestion. ${ }^{26}$

\section{Dosing and Administration}

As food significantly affects absorption, consequently reducing bioavailability, it is important that the bedtime sodium oxybate dose is taken at least two hours after eating. ${ }^{26}$ Sodium oxybate should be titrated in
Figure 1: Dosing and Administration Algorithm for Sodium Oxybate

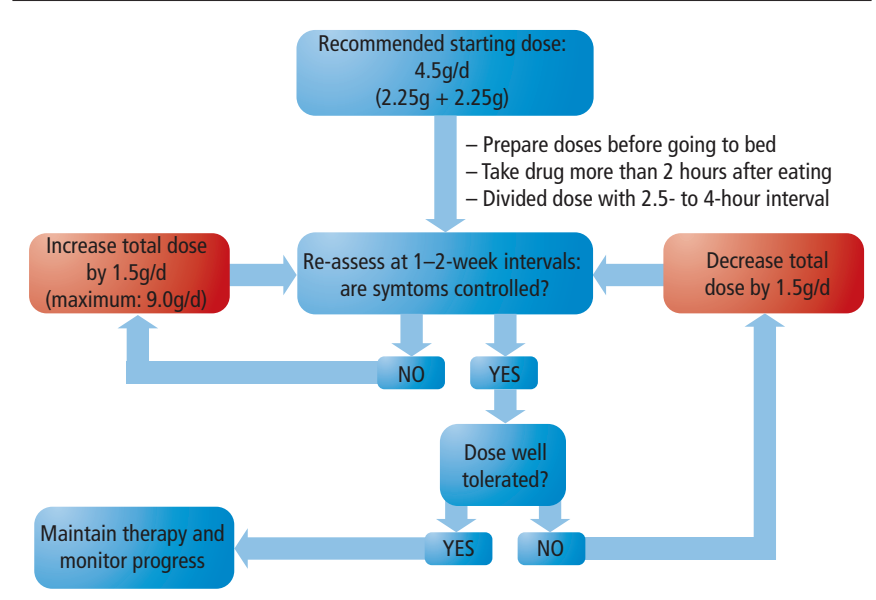

Adapted from Xyrem product SPC. ${ }^{26}$

increments of $1.5 \mathrm{~g} / \mathrm{night}$ at one- to two-week intervals to a maximum dose of $9 \mathrm{~g} / \mathrm{night}$ in order to achieve full benefit on daytime symptoms (see Figure 1). This gradual increase in dose can help to minimise the possible side effects. If poorly tolerated and troublesome side effects occur, the total dose may be decreased by $1.5 \mathrm{~g} / \mathrm{night}$ to a minimum of $3 \mathrm{~g} / \mathrm{night}$; however, the total nightly dose should always remain within the range of 3-9g/night.26 Under clinical conditions titration may occasionally be faster. If necessary, titration can be performed under direct supervision with the sodium oxybate titrated at a rate of $1 \mathrm{~g} /$ night to reach the optimal dose in three to four days. This fast titration schedule, which may be useful for patients with severe EDS, has not produced any serious adverse events compared with the usual titration rate. For the treatment of cataplexy, it may be best to perform the sodium oxybate titration in the outpatient clinic, as patients need to be at home in their usual environment to trigger typical cataplexy. In this situation, titration should be performed at one- to two-week intervals with close monitoring of response. The aim of treatment is to achieve an optimal dose that produces maximal alleviation of symptoms. The most effective dose is 9g/night; however, patients will occasionally develop unacceptable side effects, so will require a lower dose. Rarely, some patients, particularly those with a high rate of metabolism, may need an even higher dose than $9 \mathrm{~g} /$ night. Due to inter-individual differences in half-life, some patients may require multiple evenly dispersed doses of sodium oxybate during the night (three or even four doses for some patients). On the other hand, some patients have difficulty waking up to an alarm clock, or wake too close to their usual final wake-up time in the morning to be able to take the second dose. These patients can do well with a single bedtime dose; however, in general it is preferable to use two divided doses at night.

There is a clear dose response for cataplexy efficacy, with a maximum of $9 \mathrm{~g} / \mathrm{night}$. The median change in the number of cataplexy attacks per week was $69 \%$ with $9 \mathrm{~g} / \mathrm{night}$ after four weeks of treatment in one study, ${ }^{15}$ and $84.7 \%$ at $9 \mathrm{~g} / \mathrm{night}$ after eight weeks in another study. ${ }^{16}$ The improvement in cataplexy starts within the first two weeks, but further improvement occurs between two and four weeks after starting treatment..$^{15}$ It is important to avoid early withdrawal of sodium oxybate within the first two weeks because of lack of efficacy, as benefits may be seen first during weeks two to four. There is also an efficacy dose effect of sodium oxybate on EDS, as measured by the ESS. There is a significant effect of sodium oxybate at $6 \mathrm{~g} / \mathrm{night}$, with further improvement at $9 \mathrm{~g} /$ night. $^{20}$ Some patients at $9 \mathrm{~g} / \mathrm{night}$ can 
reach a normal range on the ESS. As for cataplexy, effects of sodium oxybate on EDS are maintained in long-term studies. ${ }^{17}$ The combination of sodium oxybate and modafinil has been shown to produce further improvement than with sodium oxybate alone on EDS, Clinical Global Improvement-Changes scale (CGI-C) and MWT. ${ }^{24}$ In the German experience, $26 \%$ of patients were treated with sodium oxybate alone and $74 \%$ by a combination of sodium oxybate plus modafinil, antidepressants or stimulants. The combination of modafinil and sodium oxybate is a preferred treatment option when sodium oxybate alone produces an inadequate effect on EDS. However, in patients on modafinil who start on sodium oxybate, an increase of the sodium oxybate dose with a gradual decrease of modafinil should be undertaken. No tolerance of sodium oxybate on EDS has been demonstrated. With regard to disturbed nocturnal sleep, sodium oxybate at $9 \mathrm{~g} / \mathrm{night}$ increased stage 3 and 4 sleep duration, increased mean delta power during sleep ${ }^{19}$ and reduced the total number of awakenings and duration of wakefulness after sleep onset (Black, unpublished observation). When disturbed nocturnal sleep is a major symptom, sodium oxybate is the best option to increase quality of sleep.

Sodium oxybate is the only available medication effective for the three main symptoms of narcolepsy: cataplexy, EDS and disturbed nocturnal sleep. The best improvement of QoL, as measured by the FOSQ, was reached with 9g/night sodium oxybate. ${ }^{24}$ To ensure the best efficacy from sodium oxybate treatment, the patient needs to be carefully informed about the clinical features of this new treatment, and understand the medical and psychosocial impact of narcolepsy.

\section{Side Effects}

The most commonly reported adverse drug reactions are dizziness, nausea and headache, all occurring in $10-20 \%$ of patients. Other side effects include nervousness, irritability, tremulousness, anorexia, weight loss and nocturnal eneuresis. ${ }^{26}$ In the German experience, only $12 \%$ of patients reported side effects. Difficulty in waking up in the morning, dry eyes, nausea, nervousness, constipation, sweating and temporary and unique extreme dizziness have been reported. Nocturnal enuresis, vomiting, confusion or sleepwalking were not observed in the German experience. Of particular interest was the development of unexpected beneficial effects: an improvement in depressive mood was observed in a significant proportion of patients. No published data are available on the management of side effects. The current opinion of experienced clinicians is to temporarily lower the total dose of sodium oxybate and, in most cases, the side effects disappear. After a given time at a lower dose, up-titration of the dose to reach optimal beneficial effects may be carried out, usually without a return of the side effects. In the specific case of nocturnal enuresis, either the amount of the first or the second dose could be adjusted according to the time of onset of the enuresis. Some patients dislike the salty taste of the sodium oxybate solution. A useful tip is to add some flavouring, such as mint or fruit. However, dilutants that alter the acidity of the solution, such as orange juice, should be avoided. No withdrawal symptoms have been observed.

\section{Contraindications and Special Warnings}

Sodium oxybate is contraindicated in patients with hypersensitivity to sodium oxybate or to any of the solution constituents, patients with succinic semialdehyde dehydrogenase deficiency, patients treated with opioids or barbiturates and pregnant, lactating or nursing women. ${ }^{26}$ It also seems reasonable to be cautious in any patient who needs to get up during the night to attend to a child or for other reasons, such as attending to work-related night-time telephone calls. Care should be taken in the use of sodium oxybate in the elderly, where the potential risk of falls at night is a particular concern, due to the limited experience in this population. ${ }^{26}$ Sodium oxybate is the sodium salt of GHB, a CNS depressant with abuse potential. There have been case reports of dependence after illicit use of GHB at frequent repeated doses (18-250g/day). While there is no evidence of dependency in patients taking sodium oxybate at therapeutic doses, this possibility cannot be excluded. ${ }^{26}$ Patients should be warned against the use of alcohol in conjunction with sodium oxybate as it may result in potentiation of the CNS-depressant effects of sodium oxybate. ${ }^{26}$ If alcohol is taken in the evening, it is generally recommended that the first dose of sodium oxybate be avoided.

Use of sodium oxybate for narcolepsy with cataplexy associated with obstructive sleep apnoea syndrome (OSAS) has been reported in one study (data on file). There was a mean improvement in the apnoea/hypopnoea index, although three patients had significant oxygen desaturation, which could not have been predicted. Therefore, clinicians should avoid using sodium oxybate for narcolepsy with cataplexy in patients with untreated OSAS. Narcolepsy patients with treated OSAS should be closely monitored during treatment with sodium oxybate. Patients taking sodium oxybate will have an additional daily intake of sodium that ranges from $0.75 \mathrm{~g}$ (for $4.5 \mathrm{mg} / \mathrm{night}$ ) to $1.6 \mathrm{~g}$ (for $9 \mathrm{~g} / \mathrm{night}$ ). It is recommended that dietary sodium intake be reduced in the management of patients with heart failure, hypertension or compromised renal function. ${ }^{26}$ If necessary, diuretics can be prescribed to reduce sodium levels.

\section{Conclusion}

Sodium oxybate in clinical trials has demonstrated efficacy on all major symptoms of narcolepsy, with an acceptable side effect profile. The clinician has to manage patients suffering from narcolepsy with cataplexy whose responses to treatment may vary. The recommendations given here from clinicians experienced in the use of sodium oxybate should be helpful for those unfamiliar with its use.
1. The International Classification of Sleep Disorders: Diagnostic and Coding Manual, second edition, Westchester, Illinois: American Academy of Sleep Medicine, 2005.

2. Black JE, Brooks SN, Nishino S, Semin Neurol, 2004; 24(3):271-82

3. Keam S, Walker MC, Curr Opin Neurobiol, 2007; 20:699-703.

4. Nishino S, J Clin Psychiatry, 2007;68(Suppl. 13):9-15.

5. Overeem S, Lammers GJ, Sleep Med Rev, 2008;12:95-107.

6. Nishino S, Clin, 2006;1:47-61.

7. Billiard M, Bassetti C, Dauvilliers Y, et al., Eur I Neurol, 2006;13:1035-48.

8. Dauvillier $Y$, Arnulf I, Mignot E, Lancet, 2007;369:499-511

9. Daniels E, King MA, Smith IE, Shneerson JM, J Sleep Res,
2001:10:75-81.

10. Benca RM, J Clin Psych, 2007;68(Suppl. 13):5-8.

11. Morgenthaler TI, Kapur VK, Brown T, et al., Sleep, 2007;30(12):1705-11.

12. Pardi D, Black JE, Future Neurology, 2006;1:721-35.

13. Benavides J, Rumigny JF, Life Sci, 1982;30:953-61.

14. Szabo ST, Gold MS, Goldberger BA, Blier P, Biol Psychiatry, 2004;55:934-9.

15. The US Xyrem ${ }^{\circledR}$ Multicenter Study Group, Sleep, 2002;25:42-9.

16. The Xyrem ${ }^{\circledR}$ International Study Group, Sleep Med, 2005;6: $415-21$.

17. US Xyrem ${ }^{\circledR}$ Multicenter Study Group, Sleep, 2003;26:31-5.

18. US Xyrem ${ }^{\circledR}$ Multicenter Study Group, Sleep Med, 2004;5:119-23.
19. Mamelak M, Black J, Montplaisir J, et al., Sleep, 2004:27: 1327-34.

20. The Xyrem ${ }^{\circledR}$ International Study Group, J Clin Sleep Med, 2005:1:391-7.

21. Black J, Houghton WC, Sleep, 2006;29:939-46.

22. Data on file (Study SXB-15), UCB Pharma Ltd.

23. Data on file (Study SXB-6), UCB Pharma Ltd.

24. Weaver TE, Cuellar N, Sleep, 2006;29:1189-94.

25. Data on file (Xyrem ${ }^{\circledR}$ Summary of Clinical Safety), UCB Pharma Ltd.

26. Xyrem ${ }^{\circledR}$ product SPC 